\title{
Uso de la Acupuntura y Moxibustión en el manejo de Parálisis Cerebral Tetraparesica Discinética. Reporte de un caso clínico
}

\author{
Marco Vieira ${ }^{1}$; Filomena Paulo ${ }^{1}$; Ana Francisco ${ }^{2}$; Conceição Martins ${ }^{1}$ \\ (1) Escola Superior de Saúde de Viseu, Instituto Politécnico de Viseu, Portugal. \\ (2) Hospital Garcia da Horta, Almada, Portugal.
}

\begin{abstract}
Resumen
En este artículo se presenta un estudio de caso de un niño de 11 años de edad con Parálisis Cerebral Tetraparesica Discinética que fue tratado con la Acupuntura y la Moxibustión, junto con la terapia convencional en hospital.

Se desea evaluar cómo la Acupuntura-Moxibustión puede ser ventajosa en el manejo de pacientes con parálisis cerebral.

Se hicieron tratamientos con Acupuntura y Moxibustión de manera continua a lo largo de más de 3 meses. Se observó una mejora progresiva y significativa en su cuadro clínico, lo que sugiere que la Acupuntura y la Moxibustión pueden ser una combinación eficaz de tratamiento adyuvante para Parálisis Cerebral Tetraparesica Discinética.

Palabras-clave: Acupuntura; Moxibustión; Parálisis Cerebral Infantil
\end{abstract}

\section{Abstract}

This article presents a case study of a boy 11 years old with Cerebral Palsy Tetraparesis Dyskinetic that was treated with Acupuncture and Moxibustion, along with conventional therapy in hospital.

It is intended to verify how the Acupuncture-Moxibustion can be advantageous in the cares of patients with cerebral palsy.

Continuous treatments of Acupuncture and Moxibustion were performed over 3 months. There was a progressive improvement in their clinical picture, which suggests that Acupuncture and Moxibustion can be an effective combination of adjuvant treatment for Cerebral Palsy Tetraparesis Dyskinetic.

Keywords: Acupuncture; Moxibustion; Cerebral palsy

\section{Ficha de identificación}

Nombre: DS

Sexo: masculino

Edad: 11 años

Estatura: $1,55 \mathrm{~cm}$

Peso: $51 \mathrm{~kg}$

Lugar de residencia: Portugal desde los 10 meses. 


\section{Antecedentes personales no patológicos}

Producto de embarazo gemelar prematuro 7 meses de gestación.

Es el tercero embarazo de su madre.

Ha recibido su esquema de inmunizaciones normales.

Habita con sus padres, cuenta con todos los servicios higiénicos dietéticos.

\section{Antecedentes personales patológicos}

Producto de embarazo gemelar prematuro, tuvo APGAR al nacer de 7, Peso de $1645 \mathrm{gr}$, Longitud de $41 \mathrm{~cm}$, Perímetro Cefálico de $30 \mathrm{~cm}$.

Padeció de cuadros de faringoamigadlitis de repetición lo que ha ameritado el tratamiento con antibióticos.

Crisis convulsivas tipo tonicoclónica controladas y tratadas con Keppra y

Diazepam.

Esofagitis derivada de ERGE.

Funduplicatura mayo 2010 sin complicaciones.

\section{Estado actual}

Inicia su padecimiento cuando sus padres se percataron que no podía sostener la cabeza a los 3 meses de nacido, lo que motivo consulta con su médico pediatra y posteriormente con su neurólogo que a los 6 meses se diagnosticó como tetraparesia discinética.

Desde entonces ha recibido tratamiento a base de terapia física y rehabiltación,

\section{Evaluación Física}

Se observa a niño con un crecimiento menos a su edad cronológica, orientado en la esfera física, mental y emocional, que puede sostener la cabeza, los movimientos del hombro se encuentran limitados en su abducción del hombro, flexión y extensión del codo, muñeca, dedos de la mano, cadera, rodillas, tobillos y dedos de los pies, se observan movimientos discinéticos en los músculos de las extremidades cuando el paciente intenta hacer movimientos voluntarios. Controla la deglución con dificultad, controla los esfínteres, hay espasticidad de los músculos aductores, puede sentarse, pero no puede mantenerse en pie.

Tiene importantes limitaciones, nunca tuvo la marcha autónoma, no puede soportar por sí solo, es totalmente dependiente de la realización de sus actividades y la vida cotidiana. 


\section{Diagnósticos Clínicos}

Parálisis Cerebral tipo tetraparesia discinética de etiología desconocida; distonía generalizada y el reflujo gastroesofágico post-grado de estado con esofagitis III, habiendo sido realizada fundoplicatura en mayo de 2010.

\section{Terapeutica Farmacológica:}

- Diazepam; Clonidina; Levetiracetam; Baclofeno; Tri-hexifenidilo; Hidrato cloral (tomarse sólo en situaciones SOS); Esomeprazol; Lactulose. [2]

\section{Internamientos}

Después de varias hospitalizaciones por distonía generalizada de empeoramiento progresivo, se somete a diversos ajustes terapéuticos y se decide la colocación de la estimulación cerebral profunda [2] en noviembre de 2015. Después de la intervención no si plantea mejorar su estado clínico, con un empeoramiento progresivo.

En enero de 2016, presenta, muy delgado, con anorexia, tienen dificultad para masticar y tragar, con pérdida de peso, estado de ánimo depresivo, la comunicación verbal muy limitada y menos perceptible, disminución de la producción de orina, sudoración severa durante las convulsiones, estreñimiento, ataques diarios distónicos de gran intensidad con la toma de la medicación en necesidad SOS, 2 a 3 veces por día

Parece ser que las distonías se producen debido fundamentalmente a causa emocional, cuando se despierta, cuando siente la ausencia de la madre, cuando está alterado o cuando está preocupado por algo, a la hora de comer. [3] Tuvimos 2 observaciones de la psiquiatría infantil.

Con el consenso del equipo multiprofesional decidieron, en febrero de 2016, iniciar un enfoque complementario con sesiones de Acupuntura para promover su estabilidad física y emocional para que pueda promover su regreso a casa.

\section{Diagnóstico y Etiología en la Medicina China}

A la exploración física se encuentra, un niño adelgazado en sus extremidades, reporta apetito reducido, hay estreñimiento, friolento, enfermizo, sueño normal, tendencia a la depresión, distensión abdominal, dolor y debilidad de las rodillas, cuerpo de la lengua rosado, pequeña, pastosa, saburra blanca gruesa, pulso fuerte y cuerda.

Parálisis Cerebral, en la diferenciación de la medicina china, puede ser debido a los siguientes síndromes: "Vacío de Jing (esencia) de los riñones", "Vacío de Qi (energía) y Xue (sangre)" o "Trauma o lesión en el nacimiento".

D. S. tenía un "Vacío de Jing de los Riñones." Del Jing (esencia) dependen la médula y el cerebro, llamado en Medicina China "El Mar de Médulas". El Jing 
es de naturaleza Yin, si está en Vacío lleva un síndrome de " Vacío del Yin de Riñón " que conduce a un "Vacío de Yin de Hígado", ya que riñones y hígado tienen el mismo origen. El cuadro de "Vacío de Yin de Riñón" provoca síntomas tales como pérdida de peso, anorexia, la comunicación verbal limitada, la reducción de la orina y la sudoración intensa. El descenso del Yin de Hígado conduce a un aumento del Hígado Yang que puede en fases más agudas convertirse en "Subida del Fuego de Hígado " o "Yang de Hígado se convierte en Viento", que pueden causar síntomas tales como irritabilidad, ansiedad, depresión, ataques distónicos y convulsiones, opistótonos y rigidez, así como estreñimiento severo. [4]

\section{Principios de Tratamiento}

Para los cambios en el Hígado fue seguido el principio de "Tonificar Yin y Xue del Hígado y apaciguar el Viento."

A nivel renal, el principio fue el siguiente, "Consolidación de los Riñones, fortalecer el Mar de Médulas y reconstruir la Esencia". [4]

\section{Plan de Tratamiento}

A lo largo de las sesiones (y alternativamente) eran punturados los siguientes puntos: Yintang (M-HN-3), Baihui (VC20), Zhongwan (VC12), Danzhong (VC17), Houxi (ID3), Quchi (IG11) Yinlingquan (BP9), Sanyinjiao (BP6), Zusanli (E36), Taichong (H3), Fengchi (VB20), Yanglingquan (VB34), Kunlun (V60), Taixi (R3), Shaoshang (P11). [5][6]

Comenzó a acercarse a la acupuntura con 4 sesiones, una vez al día, y más tarde llevó a cabo sesiones de 2 veces a la semana.

\begin{tabular}{|l|l|}
\hline \multicolumn{2}{|l|}{ Acupuntos seleccionados } \\
\hline Pontos: & Função: \\
\hline $\begin{array}{l}\text { Yintang (M-HN-3), Baihui } \\
\text { VC20), Fengchi (VB20) }\end{array}$ & $\begin{array}{l}\text { Tratan la dolor de cabeza, aclaran y calman la } \\
\text { mente, descienden lo Yang del Hígado, } \\
\text { desbloquean os meridianos. }\end{array}$ \\
\hline Sanyinjiao (BP6), & $\begin{array}{l}\text { Vigoriza Xue (sangre); Armoniza el Bazo, el } \\
\text { Hígado y los Riñones. }\end{array}$ \\
\hline Zusanli (E36) & Armoniza el Estómago y tonifica el Bazo. \\
\hline Taixi (R3) & Tonifica el Yin de Riñones. \\
\hline Taichong (H3) & $\begin{array}{l}\text { Mueve el Qi (energía) y el Xue (sangre); Dispersa } \\
\text { el Yang de Hígado. }\end{array}$ \\
\hline
\end{tabular}




\begin{tabular}{|l|l|}
\hline Zhongwan (VC12) & $\begin{array}{l}\text { Vigoriza el Qi (energía) y el Xue (sangre); Punto } \\
\text { Hui de las Vísceras (Fu). }\end{array}$ \\
\hline Danzhong (VC17) & Armoniza el Corazón; Calma el Shen (espirito). \\
\hline Houxi (ID3) & $\begin{array}{l}\text { Punto de apertura del Vaso Gobernador; Disminuí } \\
\text { la subida del Calor al cerebro. }\end{array}$ \\
\hline Quchi (IG11) & Libera el exceso de Yang. \\
\hline Yinlingquan (BP9) & $\begin{array}{l}\text { Armoniza la circulación de los JinYe (líquidos } \\
\text { orgánicos). }\end{array}$ \\
\hline Yanglingquan (VB34) & $\begin{array}{l}\text { Calma el Yang del Hígado y Vesicula Biliar; Punto } \\
\text { Huide los músculos y tendones. }\end{array}$ \\
\hline Kunlun (V60) & $\begin{array}{l}\text { Calma el dolor; Drena el Calor del Merid. da } \\
\text { Vejiga; Relaja los músculos. }\end{array}$ \\
\hline Shaoshang (P11) & $\begin{array}{l}\text { Dispersa el Viento del Hígado; Tonifica la } \\
\text { circulación de la Energía Ying (nutricia), } \\
\text { vigorizando los músculos. }\end{array}$ \\
\hline
\end{tabular}

Se utilizarán agujas $0,25 \mathrm{~mm} \times 25 \mathrm{~mm}$.

Manipulación: tonificación sedación equilibrada (ping bu ping xie).

A partir de la cuarta sesión se agregó Moxibustión en puro a lo largo de los canales de Estómago, de Bazo, de la Vesícula Biliar, Vaso Concepción y Vaso Gobernador.

Manipulación: moxibustión a favor de la circulación del Qi del canal.

\section{Desarrollo Clínico}

Después de 8 sesiones de Acupuntura-Moxibustión, parece tener una mejora progresiva en su condición clínica con:

- Más períodos de relajación del cuerpo, lo que permite la administración más de espacio de la terapia de SOS cada 24 a 48 horas

- Algunas distonías eran posible revertir con medidas de posicionamiento y de autocontrol. [8]

- Mejora del apetito (empieza a pedir comida e refiriendo sede)

- El aumento de peso de $4 \mathrm{~kg}$ (alrededor de 1,5 kg en 4 semanas).

- Mejora del estado de ánimo (vuelve a sonreír y jugar).

- Mejora de su comunicación (expresión verbal más notable).

- Regulación del tránsito intestinal y de vejiga.

Tiene alta clínica un mes después del inicio de las sesiones.

Después de la alta clínica se mantuvo sesiones de acupuntura en casa dos veces por semana, siguiendo el mismo plan de tratamiento.

Actualmente queda en casa ya hay mes y medio, con el apoyo domiciliario de cuidados continuados y sesiones de Acupuntura 2 veces a la semana,

pudiendo verificarse: 
- Control de la crisis de distonía (que ocurre principalmente cuando el despertar con la necesidad de la administración terapéutica en SOS).

- Durante el día permanece relajado, pasando el día en una silla de ruedas.

- La mejora de su estado de ánimo es feliz de estar de vuelta a casa y estar con la familia.

- Muestra disposición a volver a la escuela en la que ya se integró.

- Come bien, bebe bien, refiere apetito.

- Tránsito intestinal y vesical restaurado (evacua todo los días o en 2 días y orina 3 veces al día).

\section{Discusión y Conclusion}

La Parálisis Cerebral no es una enfermedad aislada e independiente, incluye muchos de los trastornos de movimiento del sistema nervioso central resultante de lesiones cerebrales que tienen muchas causas diferentes. En la actualidad no existe un método específico de tratamiento para la Parálisis Cerebral. [1][2] En conclusión, este caso sugiere que la Acupuntura y la Moxibustión pueden ser una combinación eficaz de tratamiento adyuvante para la Parálisis Cerebral Tetraparesica Discinética. [8]

Aunque esto es sólo un caso clínico de tratamiento y conclusiones de éxito no se puede generalizar a partir de un solo caso, sugiere que sería interesante realizar más investigaciones sobre este tema.

\section{Bibliografia}

[1] Miller, F., Bachrach, S.J. (1995). Cerebral Palsy: A Complete Guide for Caregiving. Baltimore: The John Hopkins University Press

[2] Dan, B., Mayston, M., Paneth, N., Rosenbloom, L. (2015). Cerebral Palsy:

Science and Clinical Practice (Clinics in Developmental Medicine). London: Mac Keith Press

[3] Prigatano, G.P. (1999). Principles of Neuropsychological Rehabilitation. Oxford: Oxford University Press

[4] Vieira, M. (2015).Compendium of Syndromes in Chinese Medicine. CreateSpace Independent Publishing Platform

[5] Ross, J. (1995). Acupuncture Point Combinations: The Key to Clinical Success. London: Churchill Livingstone

[6] Vieira, M. (2015). Breviário de Medicina Tradicional Chinesa. Lisboa: Ed.

Causa das Regras

[7] Sharma, K.N. (2013). Acupuncture for Cerebral Palsy Simplified: An

Illustrated Guide. CreateSpace Independent Publishing Platform; III edition

[8] Martin, S. (2006). Teaching Motor Skills to Children with Cerebral Palsy and

Similar Movement Disorders: A Guide for Parents and Professionals. Bethesda:

Woodbine House, Inc. 